\title{
Meta-analysis on the role of exercise in the treatment of constipation
}

\author{
Thomas Weiser, PhD, Sabine Landes, PhD
}

Current guidelines recommend life-style adjustment (including more exercise) as the first measure for treating constipation. This meta-analysis investigated the influence of exercise therapy on the symptoms of patients with chronic constipation. About half of participants in a exercise programme lasting several weeks experienced a positive effect on their symptoms. Studies with the laxatives bisacodyl and sodium picosulfate showed that these generally alleviated symptoms even within the first day of treatment.

c onstipation is a health disorder that significantly diminishes the quality of life of those affected [4] and has considerable economic implications (e.g. through visits to the doctor and sick leave). Successful treatment of constipation is therefore extremely worthwhile.

Guidelines on the treatment of (chronic) constipation recommend a multistage treatment algorithm. The first stage consists of lifestyle changes (increased intake of fluids, more exercise, eating more fibre); the second stage, OTC laxatives such as bisacodyl, sodium picosulfate and macrogol, whilst the third stage involves prescription-only products $[1,4]$.

A number of studies have investigated the influence of exercise on the digestion of patients with constipation and these studies have now been summarised in a meta-analysis by Gao et al. [2]. The authors identified 9 studies (with a total of 680 patients) that examined the effect of exercise training on constipation. The comparator groups received auricular acupuncture, Maziren tablets (traditional Chinese medicine), education about constipation, or no particular treatment. The sports activities varied widely, but were mostly aerobic exercises (Qigong, Baduanjin (both from Asian culture) or (fitness) walking); only one study investigated the effects of strength training.

Training consisted of 60 to 420 minutes of exercising per week for between 4 and 24 weeks. The relative risk (risk ratio; RR) of relief from the constipation was the parameter chosen to assess the effects. So what did the analysis show?

Despite the very heterogeneous nature of the exercise training, the result was nevertheless quite clear: relief from constipation had improved in patients who had completed exercise training compared to the comparator groups. However, this effect was only observed after aerobic training (where the $\mathrm{RR}$ was 2.42 in favour of the exercising groups). In contrast, anaerobic training had no effect (RR of 0.85 ). If the two forms of exercising were combined, then there was still an RR of 1.97. Exercise training is thus apparently helpful in relieving constipation, and aerobic exercising for at least 140 minutes per week showed the best effects. Some of the studies also assessed parameters such as vitality, well-being and quality of life and here too, exercise training had a positive effect.

So what are the implications of these results for everyday practice? What do the measured RRs actually mean? Let's take an example: Supposing 10 patients suffer from constipation, only 5 will get relieve from exercise training, although they have exercised between 4 and 24 weeks (see Figure 1, left panel).

According to the current guidelines [1,4], first stage treatment of constipation comprises lifestyle changes (excersise, hydration, fibre), and if this does not work, laxatives (like macrogol, bisacodyl, sodium picosulfate). Two clinical studies (in which the drugs bisacodyl or sodium picosulfate were compared with a placebo), showed that both these drugs were effective, safe and well-tolerated in the treatment of constipation. For example, approx. $70 \%$ of patients who took sodium picosulfate and $90 \%$ of those who used bisacodyl were able to have a bowel motion within the first 24 hours after ingestion [ 3,5 as well as "data on file", Sanofi Aventis Deutschland GmbH; Figure 1, right panel], and the relief of constipation symptoms was recorded throughout the entire duration of the study ( 4 weeks in each case).

Evid Self Med 2021;1:210001 | https://doi.org/10.52778/efsm.21.0001

Affiliation/Correspondence: Thomas Weiser, PhD, Sabine Landes, PhD, Consumer Healthcare Medical Affairs, Sanofi-Aventis Deutschland GmbH, Industriepark Hoechst, 65026 Frankfurt am Main, Germany (thomas.weiser@sanofi.com) 


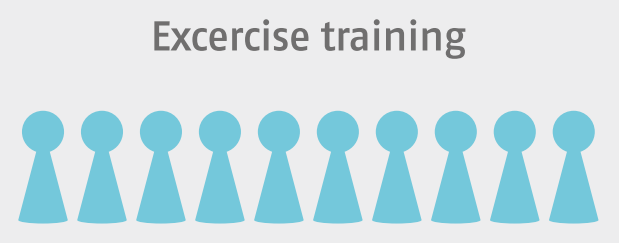

4 to 24 weeks

of training

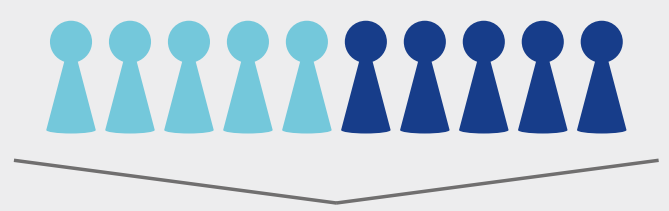

Constipation symptoms relieved in $50 \%$

\section{Bisacodyl treatment}
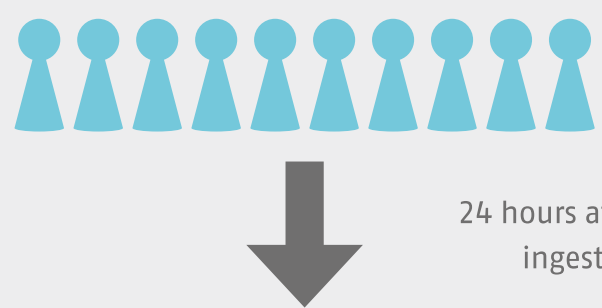

24 hours after ingestion

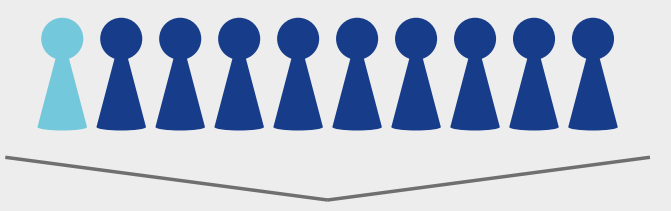

Constipation symptoms relieved in $90 \%$

Left: Exercise training for 4 to 24 weeks relieves constipation in about $50 \%$ of the exercisers. Right: Treatment with bisacodyl leads to a bowel motion within 24 hours in approx. $90 \%$ of those treated. (Data on file)

Conclusions: According to Goa [2], exercise training over several weeks has a positive effect on constipation in about half of the exercisers. Laxatives containing the drugs bisacodyl or sodium picosulfate usually relieve symptoms within the very first day after ingestion in up to $90 \%$ of patients.

\section{Literature}

1. Andresen et al. S2k Guideline for Chronic Constipation: Definition, Pathophysiology, Diagnosis and Therapy. Z Gastroenterol. 2013;51:651-672.

2. Gao R, Tao Y et al. Exercise Therapy in Patients with Constipation: A Systemic Review and Meta-Analysis of Randomized Controlled Trials. Scandinavian Journal of Gastroenterology. 2019;54:169-177.

3. Kamm MA, Müller-Lissner S et al. Oral Bisacodyl is Effective and Well-Tolerated in Patients with Chronic Constipation. Clinical Gastroenterology and Hepatology. 2011;9:577-83.

4. McCormick D. Managing Costs and Care for Chronic Idiopathic Constipation. The American Journal of Managed Care. 2019;25:S63S69.

5. Müller-Lissner S, Kamm MA et al. Multicenter, 4-Week, DoubleBlind, Randomized, Placebo-Controlled Trial of Sodium Picosulfate in Patients With Chronic Constipation; American Journal of Gastroenterology. 2010;105:897-903.
Conflict of interest: T. Weiser and S. Landes are employees of Sanofi.

Publication funded by Sanofi Aventis Deutschland GmbH.

\section{Information regarding manuscript}

Submitted on: 14.09 .2020

Accepted on: 27.12.2020

Published on: 16.08.2021 